\title{
The Mean Sea Level Equation and Its Application to the Mediterranean Sea
}

\author{
N. PINARDI \\ Department of Physics and Astronomy, Bologna University, Bologna, Italy \\ A. Bonaduce, A. NAvarra, And S. Dobricic \\ Centro EuroMediterraneo sui Cambiamenti Cimatici, Bologna, Italy \\ P. ODDO \\ Istituto Nazionale di Geofisica e Vulcanologia, Sezione di Bologna, Bologna, Italy
}

(Manuscript received 9 March 2013, in final form 2 October 2013)

\begin{abstract}
A formalism to obtain a mean sea level equation (MSLE) is constructed for any limited ocean region and/or the global ocean by considering the mass conservation equation with compressible effects and a linear equation of state. The MSLE contains buoyancy fluxes terms representing the steric effects and the mass flux is represented by surface water fluxes and volume transport terms. The MSLE is studied for the Mediterranean Sea case using a simulation experiment for the decade 1999-2008. It is found that the Mediterranean MSL tendency is made of a steric contribution that is almost periodic in time superimposed on a stochasticlike signal due to the mass balance, dominating the MSL tendency. The MSL tendency stochastic-like term is a result of the imbalance between the volume flux at Gibraltar and the area average surface water flux.
\end{abstract}

\section{Introduction}

The global mean sea level (MSL) trend has been shown to be a useful indicator of climate change and ocean heat content variability (Solomon et al. 2007). Recently, satellite altimetry analysis studies (Cazenave and Remy 2011) have reevaluated the mean sea level trend from satellite altimetry and tide-gauge records and found that thermal expansion and mass changes may be equally important contributors to the global mean sea level trend.

Theoretical investigations on the dynamics of the MSL have received relatively less attention. Greatbatch (1994) proposed a simplified version of the global MSL equation (MSLE). In this paper we formulate an MSLE that takes into account all dynamical processes internal to the oceanic water body, neglecting the glacial isostatic adjustment (Nakada and Lambeck 1987). Such an equation can be used to test different dynamical hypotheses

Corresponding author address: Department of Physics and Astronomy, University of Bologna, Viale B. Pichat 62, Bologna, Italy.

E-mail: nadia.pinardi@unibo.it and allows us to diagnose the terms responsible for the MSL tendency from ocean general circulation models (OGCMs), reanalyses, or observational datasets.

Greatbatch (1994) and Mellor and Ezer (1995) tried for the first time to define the MSLE contributing terms with different ad hoc simplifications. They succeeded in showing the importance of steric effects in determining the MSL tendency but they neglected mass changes due to the water cycle. We want to generalize their approach and write the complete MSLE for any limited region as well as the global ocean. Recently, Griffies and Greatbatch (2012) have analyzed different processes implied by the sea level equation in global climate models, but their formulation did not point out the effects of lateral boundary terms as we do in this paper.

We apply our formalism to the Mediterranean Sea case where important lateral fluxes occur at Gibraltar that add complexity to the dynamics of the MSL and we determine for the first time the different dynamical contributions to the MSL tendency in this region. We will use a 10-yr simulation dataset from an operational model of the Mediterranean Sea (Oddo et al. 2009) to diagnose and understand the balance between the steric and mass effects in this region and reconstruct the MSL for the region. 
The paper is constructed as follows: Section 2 illustrates the formalism and writes the MSLE. Section 3 evaluates the different terms in the MSLE for the Mediterranean Sea, section 4 shows the MSL reconstruction compared to satellite altimetry, and section 5 presents the conclusions.

\section{The mean sea level equation}

The sea level equation is normally obtained considering the continuity equation. In our case we make the assumption that the density is written as $\rho=\rho_{0}+\rho^{\prime}$, where $\rho_{0}$ is a constant such that $\left(\rho^{\prime} / \rho_{0}\right) \ll 1$ (ChusmanRoisin 1994) and the mass conservation is written

$$
\nabla \cdot \mathbf{u}=-\frac{1}{\rho_{0}} \frac{D \rho^{\prime}}{D t}
$$

where $(D / D t)=(\partial / \partial t)+\mathbf{u} \cdot \nabla$ and $\mathbf{u}=(u, v, w)$ is the three-dimensional velocity field. To obtain the sea level equation we need to vertically integrate Eq. (1) between the sea level $\eta(x, y, t)$ and the bathymetry $-H(x, y)$ using the kinematic and dynamic boundary conditions at the two vertical boundaries of the fluid (Apel 1987):

$$
\begin{gathered}
w_{z=\eta}=\frac{D_{2} \eta}{D t}+q_{w} \quad \text { and } \\
w_{z=-H}=-\mathbf{u}_{z=-H} \cdot \nabla H,
\end{gathered}
$$

where $\left(D_{2} / D t\right)=(\partial / \partial t)+\mathbf{u}_{z=\eta} \cdot \nabla, \mathbf{u}_{z=\eta}=\left(u_{z=\eta}, \boldsymbol{v}_{z=\eta}\right)$ is the surface velocity, $\mathbf{u}_{z=-H}=\left(u_{z=-H}, v_{z=-H}\right)$ is the bottom velocity, and

$$
q_{w}=E-P-\sum_{i} R_{i} / A_{i}-M_{G} / C .
$$

Equation (2) is the normalized freshwater flux composed of the evaporation $E$, the precipitation $P$, and the river runoff $R_{i}$, with the river cross-sectional area $A_{i}$, the freshwater runoff from glacier melting $M_{G}$, and the cross-sectional area $C$ over which the ice melting runoff occurs. The runoff and the glacier melting are considered as a surface flux but our formalism will not change if they are regarded as lateral fluxes.

Integrating Eq. (1) in the vertical we now obtain the sea level dynamic equation:

$$
\frac{\partial \eta}{\partial t}+\nabla \cdot[(H+\eta) \overline{\mathbf{u}}]=-q_{w}-\frac{1}{\rho_{0}} \int_{-H}^{\eta} \frac{D \rho^{\prime}}{D t} d z^{\prime},
$$

where $\overline{\mathbf{u}}=[1 /(H+\eta)] \int_{-H}^{\eta} \mathbf{u} d z^{\prime}$ is the barotropic velocity field. We want now to rewrite the right-hand side of Eq. (3) in a form that allows us to identify the temperature and salinity effects in the density term. We consider the following explicit form of the equation of state:

$\rho=\rho_{0}+\rho^{\prime}=\rho_{0}+\rho_{f}\left[-\alpha_{T}\left(T-T_{f}\right)+\beta\left(S-S_{f}\right)\right]$,

where $T$ is the potential temperature, $S$ is the salinity, $\alpha_{T}=-\left.\left(1 / \rho_{f}\right)(\partial \rho / \partial T)\right|_{T=T_{f}}$ and $\beta=\left.\left(1 / \rho_{f}\right)(\partial \rho / \partial S)\right|_{S=S_{f}}$ are the thermal expansion and the haline contraction coefficient respectively, $T_{f}$ and $S_{f}$ are reference potential temperature and salinity profiles, and $\rho_{f}$ is a constant reference value (Marshall and Plumb 2008). The linearized equation of state [Eq. (4)] is considered because it is widely used in sea level studies (Levitus et al. 2012) and it will allow us to rewrite the rhs of Eq. (3) in terms of buoyancy forcing.

Moreover considering $\alpha_{T}$ and $\beta$ constant, the rhs of Eq. (1) can be rewritten using the first law of thermodynamics and the salinity equation (Pedlosky 1987):

$$
\frac{D \rho^{\prime}}{D t}=\nabla \cdot\left(K_{h} \nabla \rho^{\prime}\right)+\frac{\partial}{\partial z}\left(K_{v} \frac{\partial \rho^{\prime}}{\partial z}\right),
$$

where $K_{h}$ and $K_{v}$ are the horizontal and vertical diffusivity coefficients. The surface boundary condition for Eq. (5) is

$$
\left.K_{v} \frac{\partial \rho^{\prime}}{\partial z}\right|_{z=\eta}=-B
$$

and $B$ is the scaled buoyancy flux,

$$
B=\alpha_{T} \frac{Q}{C_{w}}-\rho_{f} \beta S_{0} q_{w},
$$

where $S_{0}$ is the surface salinity field, $Q$ is the net flux of heat into the ocean (negative during night), and $C_{w}$ is the specific heat capacity of seawater.

Defining now the MSL by $\langle\eta(t)\rangle$, where the average operator is taken over a sea area $\Omega$ with open boundaries $\partial \Omega$,

$$
\langle\rangle=\frac{1}{\Omega} \iint_{\Omega} d \Omega .
$$

The final form of the MSLE is obtained, taking the horizontal average of Eq. (3) and inserting the definition of $B$ :

$$
\frac{d\langle\eta\rangle}{d t}=-\underbrace{\frac{T_{r}}{\Omega}}_{1}-\underbrace{\left\langle q_{w}\right\rangle}_{2}-\underbrace{\frac{\rho_{f}}{\rho_{0}}\left\langle\beta S_{0} q_{w}\right\rangle}_{3}+\underbrace{\frac{1}{\rho_{0}}\left\langle\alpha_{T} \frac{Q}{C_{w}}\right\rangle}_{4}-\underbrace{F_{\rho}}_{5},
$$


where

$$
\begin{aligned}
& T_{r}=\oint_{\partial \Omega} \int_{-H}^{\eta} \mathbf{u} \cdot \mathbf{n} d z d l \text { and } \\
& F_{\rho}=\frac{1}{\rho_{0} \Omega} \oint_{\partial \Omega}\left(\int_{-H}^{\eta} K_{h} \nabla \rho^{\prime} d z\right) \cdot \mathbf{n} d l .
\end{aligned}
$$

Here $T_{r}$ is the net volume transport out of the domain and $F_{\rho}$ is the normalized density flux, both positive in the outward direction.

There are five terms contributing to the MSL tendency [Eq. (9)]. Term 1 is the net volume transport that changes the mass in the domain and term 2 is the net addition or loss of water by surface processes. These two terms correspond to the mass tendency terms and they compose the MSL tendency in incompressible models. We will call them collectively the incompressible terms.

The other three terms come from the compressible part of the continuity equation and they are often referred to as steric effect terms even if they have not been written explicitly this way before. Term 3 is due to the salinity vertical flux condition and we will call it the halosteric effect term. Term 4 is the heat flux term and we will call it the thermosteric term. Term 3 is a novel form of the halosteric term while term 4 has been already written by Greatbatch (1994). Finally, term 5 is the density flux out of the domain accounting for changes of density advected in or out of the domain of interest. An alternative form of Eq. (9) can be formulated that considers still a linear equation of state but nonconstant thermal and haline coefficients; it is reported in the appendix.

If the domain is the global ocean, the boundary terms disappear and the MSLE for the global sea level $\left\langle\eta_{G}\right\rangle$ is

$$
\frac{d\left\langle\eta_{G}\right\rangle}{d t}=-\left\langle q_{w}\right\rangle-\frac{\rho_{f}}{\rho_{0}}\left\langle\beta S_{0} q_{w}\right\rangle+\frac{1}{\rho_{0}}\left\langle\alpha_{T} \frac{Q}{C_{w}}\right\rangle .
$$

Equation (10) can be used to give an estimate of the global sea level rise knowing only air-sea surface fluxes.

Mellor and Ezer (1995), comparing incompressible with compressible numerical model solutions, suggested that the full MSL can be well reconstructed by adding the incompressible to the steric contributions. Thus, we argue that the solution of the MSLE [Eq. (9)] can be obtained by solving two separate problems:

$$
\begin{aligned}
\langle\eta\rangle & =\left\langle\eta_{i}\right\rangle+\left\langle\eta_{s}\right\rangle \quad \text { then } \\
\frac{d\left\langle\eta_{i}\right\rangle}{d t} & =-\frac{T_{r}}{\Omega}-\left\langle q_{w}\right\rangle \quad \text { and }
\end{aligned}
$$

$$
\frac{d\left\langle\eta_{s}\right\rangle}{d t}=-\frac{\rho_{f}}{\rho_{0}}\left\langle\beta S_{0} q_{w}\right\rangle+\frac{1}{\rho_{0}}\left\langle\alpha_{T} \frac{Q}{C_{w}}\right\rangle-F_{\rho},
$$

where $\left\langle\eta_{i}\right\rangle$ is the MSL component due to incompressible dynamics and $\left\langle\eta_{s}\right\rangle$ is the steric part. Our subdivision considers that the $T_{r}$ flux is weakly dependent on the steric effects (i.e., the $\eta_{s}$ component of sea level). In the next section we will study the terms contributing to Eq. (9) for the Mediterranean Sea.

\section{The MSLE balance of terms in the Mediterranean Sea}

The Mediterranean Sea is a semienclosed, antiestuarine basin (Pinardi and Masetti 2000) that has two lateral openings, one at the Gibraltar and the other at the Dardanelles Strait. We use here a high-resolution OGCM implemented in the Mediterranean Sea and validated for sea level variability (Oddo et al. 2009). The OGCM considers the incompressible and Boussinesq approximations so that the steric terms are evaluated a posteriori from the model output. In our calculations we have considered that $\alpha_{T}=2.2 \times 10^{-4}$ and $\beta=7.3 \times$ $10^{-4}$ computed as an average over the model volume and $\rho_{f}=\rho_{0}=1025 \mathrm{~kg} \mathrm{~m}^{-3}$.

In Fig. 1 we show all the five terms in Eq. (9): the transport at Gibraltar, the water flux, and the thermosteric term are the largest contributors followed by the halosteric term and last by the density advection term, which is several orders of magnitude smaller than all the others. We note that the Gibraltar transport and the water flux terms are approximately in phase but with opposite sign, their sum resulting into a highly varying, stochastic-like term (Fig. 1). The steric terms instead oscillate almost periodically around zero.

We believe that this balance of terms offers a new interpretation of the MSL tendency in the Mediterranean Sea: the latter is composed of the almost periodic steric component superimposed on a stochastic-like mass contribution, resulting from the imbalance between Gibraltar and the surface water flux. It is well known that the Mediterranean in the long term loses water at the surface ( $q_{w}$ is positive) and receives mass from the Atlantic Ocean to balance the loss (i.e., $T_{r}$ is negative). If a steady state is achieved in the long-term mean, these two fluxes should perfectly balance but annually and seasonally this balance is not attained and the incompressible terms will give rise to an MSL tendency. The steric terms, on the other hand, arise from the variation of the buoyancy fluxes at the seasonal time scales and have important interannual anomalies that contribute also to a long-term mean tendency. 

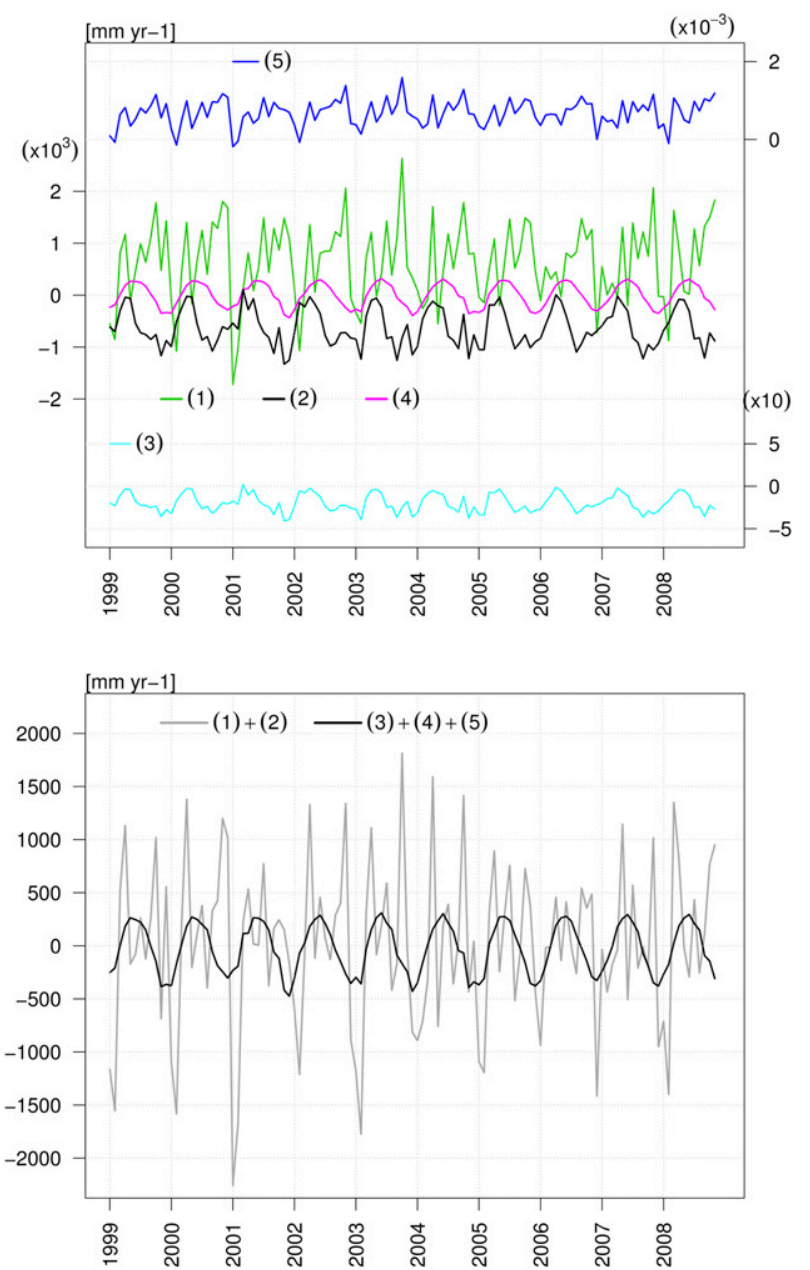

FIG. 1. The MSLE terms $\left(\mathrm{mm} \mathrm{yr}^{-1}\right)$ for the Mediterranean Sea and the period 1999-2008. (top) All the terms on the rhs of Eq. (9). (bottom) The sum of incompressible (terms 1 and 2, gray line) and steric (terms 3-5, black line) terms in Eq. (9).

If we consider the MSL tendency anomaly from a multiyear climatological mean, the result is the stochasticlike tendency anomaly shown in Fig. 2 that is clearly dominated by the incompressible terms giving rise to fluctuations with range of values between $\pm 1000 \mathrm{~mm} \mathrm{yr}^{-1}$. It is then concluded that the MSL tendency in the Mediterranean Sea is governed by a highly irregular, stochasticlike signal that will produce the MSL trend in the region. This stochastic-like term is dominated by the seasonal and yearly imbalance between the surface water flux and the Gibraltar Strait transport. The high values of the stochastic fluctuations of the MSL tendency are probably a result of the specific model implementation for the Mediterranean Sea but we expect that a stochastic-like term would be generally present in the MSL tendency of limited ocean areas because of dynamical processes that offset the balance between lateral and vertical mass fluxes.

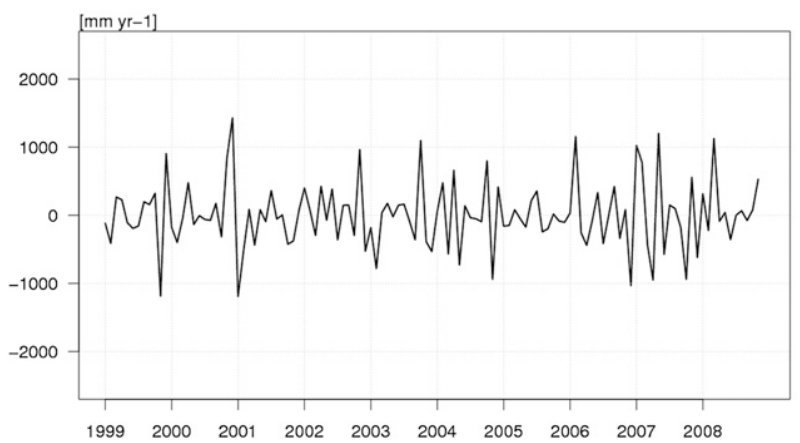

FIG. 2. The Mediterranean Sea MSL tendency anomaly; i.e., the sum of all terms in Eq. (9) with a multiyear monthly-mean term subtracted for the period 1999-2008 $\left(\mathrm{mm} \mathrm{yr}^{-1}\right)$.

\section{The MSL reconstruction in the Mediterranean Sea}

To verify that the balance of terms in Eq. (9) reproduces the observed MSL for the Mediterranean Sea, we will find a solution of the MSLE and compare it with the satellite MSL signal. The solution is obtained using Eq. (11), which means solving Eqs. (12) and (13) separately. The rhs terms of Eqs. (12) and (13) are evaluated from the OGCM output at each model time step (600s) that is also the MSLE time step for a Runge-Kutta time integration scheme.

To calibrate the numerics, the reconstructed MSL from Eq. (12) for the period 1999-2008 is compared with the MSL obtained averaging the OGCM sea level over the Mediterranean Sea area. It is found that by integrating for $10 \mathrm{yr}$ (i.e., about 525000 time steps), the numerical error produced departures of several centimeters between the two MSL solutions. Thus we considered a reinitialization of Eq. (12) from the model solution values each year, obtaining a 10 -yr average root-mean-square error for the difference between the reconstructed and the OGCM MSL of $0.47 \mathrm{~cm}$.

Following the same method explained above, Eq. (13) is restarted every year from a calculated steric MSL computed using Eq. (A4). The final solution is compared with mapped satellite anomalies shown in Fig. 3. The satellite sea level anomaly (SLA) maps are constructed using multimission along-track data up to $20 \mathrm{~km}$ from the coasts (Le Traon et al. 1998) and objectively analyzed (Ducet et al. 2000) on a $1 / 8^{\circ} \times 1 / 8^{\circ}$ resolution grid (Pujol and Larnicol, 2005). The correlation between the MSL anomalies from altimetry and our reconstruction is 0.7 and the root-mean-square error is $2 \mathrm{~cm}$, which is within the estimated error for the satellite SLA.

Uncertainties in the MSL reconstruction derive from the approximations used to write the MSLE and to the numerical method used. For a stochastic-like ordinary 


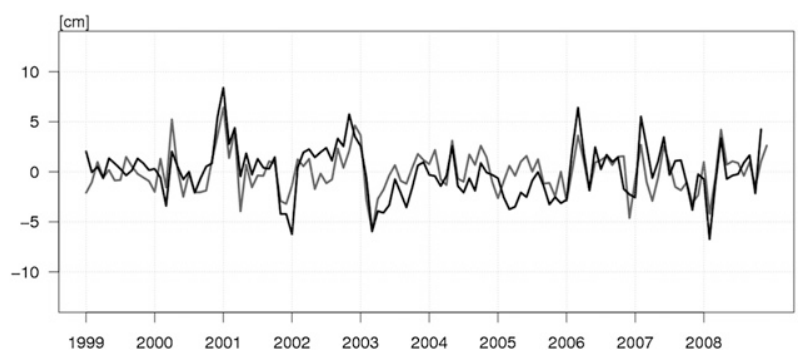

FIG. 3. The reconstructed MSL model anomalies (gray line) and the satellite altimetry anomalies (black line) obtained subtracting the 10-yr climatology $(\mathrm{cm})$

differential equation initial condition errors may propagate rapidly, causing the solution to deteriorate. On the other hand, uncertainties in the estimate of the MSL from altimetry concern the objective mapping procedure in addition to the uncertainties in atmospheric and long wavelength along-track corrections. We argue that, within the approximations of our MSLE and the satellite data mapping uncertainty, the Mediterranean Sea is well reconstructed by the terms given in Eq. (9).

For the sake of completeness, we have evaluated the MSL using Eq. (A3) and the results (not shown) are qualitatively similar to those reported above since the stochastic terms are evaluated exactly the same way in Eqs. (A3) and (9) and the steric ones are very similar.

\section{Conclusions and discussion}

In this paper we have deduced an MSLE containing both incompressible and steric processes and shown its application to the Mediterranean Sea.

The MSLE is written for the first time for any semienclosed or limited area of the World Ocean using two major approximations: 1) the density anomalies are smaller than the constant value of density and 2) the equation of state for density is linear and with constant coefficients. The latter assumption allows us to write the MSLE entirely in terms of surface buoyancy and lateral fluxes at the open sides of the limited domain. Another derivation of the MSLE is given in the appendix that removes the constant coefficient assumption and invokes a linearization of the density total derivative in the continuity equation.

The MSLE for a generic, limited area of the World Ocean is composed of five terms, two of them representing the mass fluxes at the open boundaries, so-called incompressible terms, and the others representing steric components, because of a combination of thermosteric and halosteric effects and lateral density fluxes.

Computing the terms in the MSLE for the Mediterranean Sea it is found that the MSL tendency is composed of three main terms: the two incompressible terms that provide a stochastic-like signal and the regular, almost periodic term due to steric effects. We argue that the resulting stochastic-like term, resulting from the imbalance between the lateral mass and the surface water flux, will be similar in other World Ocean, semienclosed basins, giving rise to a major contribution to the regional MSL tendency. For the Mediterranean Sea, the main contributors to the variability of the MSL tendency are the incompressible terms.

Equation (9) can be integrated using the separation of the incompressible and steric terms, giving a reconstruction of the MSL in the region of interest. It is interesting to note that the estimation of the precision required to integrate the MSLE can give some indication as to the magnitude of the uncertainties involved in simulating the MSL, at least in limited areas of the World Ocean. The MSL reconstructed from the explicit integration of the MSLE deviates significantly from the area averaged, model simulated sea level every few years, with an error of about $2-3 \mathrm{~cm}$, which can be assumed to be an estimate of the MSL uncertainty magnitude.

The formalism of this paper, evaluated for the whole Mediterranean Sea, should be applied in the future to each separate subbasin. For the incompressible part of the sea level $\left(\eta_{i}\right)$, the effects of lateral mass flux at Gibraltar will be partially balanced by the Sicily Strait transport for the western Mediterranean, while in the eastern Mediterranean they will be dependent upon the Sicily Strait mass inflow that will be balanced by the net surface water flux in that part of the basin. Furthermore, future research should formulate the MSLE in the framework of the nonlinear equation of state and should carry out the study of the MSLE dynamical balances for other World Ocean semienclosed regions.

Acknowledgments. The primary source of support for this work is the Ph.D. Program offered by the University of Bologna to Dr. Antonio Bonaduce and the computer resources offered by the Sezione di Bologna, Istituto Nazionale di Geofisica e Vulcanologia. NP particularly thanks Prof. Enzo Boschi for having suggested to start the investigations on sea level variability and having supported these activities with enthusiasm.

\section{APPENDIX}

\section{Alternative Formulation of the MSLE}

Equation (9) is derived considering a linear equation of state with constant coefficients that allows us to write thermosteric and halosteric effects in terms of surface buoyancy fluxes. Steric effects are normally defined 
considering temperature and salinity anomalies and space- and time-varying $\alpha_{T}$ and $\beta$ coefficients and here we will recover the more commonly used form. Starting from a linearized form of the sea level Eq. (3),

$\frac{\partial \eta}{\partial t} \simeq-\nabla \cdot[(H+\eta) \overline{\mathbf{u}}]-q_{w}-\frac{1}{\rho_{0}} \frac{\partial}{\partial t} \int_{-H}^{0} \rho^{\prime} d z^{\prime}$, and using the density Eq. (4) with space and time varying coefficients, $\check{\alpha}_{T}$ and $\check{\beta}$,

$$
\rho=\rho_{0}+\rho^{\prime}=\rho_{0}+\rho_{f}\left[-\check{\alpha}_{T}\left(T-T_{f}\right)+\check{\beta}\left(S-S_{f}\right)\right],
$$

we obtain a new approximate form of the MSLE:

$$
\frac{d\langle\eta\rangle}{d t} \simeq \underbrace{\frac{T_{r}}{\Omega}}_{1}-\underbrace{\left\langle q_{w}\right\rangle}_{2}-\underbrace{\frac{\rho_{f}}{\rho_{0}} \frac{d}{d t}\left\langle\int_{-H}^{0} \check{\beta}\left(S-S_{f}\right) d z^{\prime}\right\rangle}_{3^{\prime}}+\underbrace{\frac{\rho_{f}}{\rho_{0}} \frac{d}{d t}\left\langle\int_{-H}^{0} \check{\alpha}_{T}\left(T-T_{f}\right) d z^{\prime}\right\rangle}_{4^{\prime}} .
$$

In this alternative MSLE, the steric terms of Eq. (9) are replaced by the tendency of the vertical integral of the temperature and salinity anomalies. If we indicate the steric component of sea level with

$\left\langle\eta_{s}\right\rangle=\frac{\rho_{f}}{\rho_{0}}\left\langle\int_{-H}^{0}\left[-\check{\alpha}_{T}\left(T-T_{f}\right)+\check{\beta}\left(S-S_{f}\right)\right] d z^{\prime}\right\rangle$,

we obtain another useful form of Eq. (A3):

$$
\frac{d}{d t}\left(\langle\eta\rangle+\left\langle\eta_{s}\right\rangle\right)=-\frac{T_{r}}{\Omega}-\left\langle q_{w}\right\rangle .
$$

\section{REFERENCES}

Apel, J., 1987: Principles of Ocean Physics. International Geophysical Series, Vol. 38, Academic Press, 634 pp.

Cazenave, A., and F. Remy, 2011: Sea level and climate: Measurements and causes of changes. Wiley Interdiscip. Rev.: Climate Change, 2, 647-662, doi:10.1002/wcc.139.

Chusman-Roisin, B., 1994: Introduction to Geophysical Fluid Dynamics. Prentice Hall, 320 pp.

Ducet, N., P.-Y. Le Traon, and G. Reverdin, 2000: Global highresolution mapping of ocean circulation from TOPEX/ Poseidon and ERS-1 and -2. J. Geophys. Res., 105 (C8), 19477-19498.

Greatbatch, R. J., 1994: A note on the representation of steric sea level in models that conserve volume rather than mass. J. Geophys. Res., 99 (C6), 12767-12 771.

Griffies, S. M., and R. J. Greatbatch, 2012: Physical processes that impact the evolution of global mean sea level in ocean climate models. Ocean Modell., 51,37-72, doi:10.1016/ j.ocemod.2012.04.003.

Le Traon, P., F. Nadal, and N. Ducet, 1998: An improved mapping method of multisatellite altimeter data. J. Atmos. Oceanic Technol., 15, 522-534.

Levitus, S., and Coauthors, 2012: World ocean heat content and thermosteric sea level change $(0-2000 \mathrm{~m}), 1955-2010$. Geophys. Res. Lett., 39, L10603, doi:10.1029/2012GL051106.

Marshall, J., and R. Plumb, 2008: Atmosphere, Ocean, and Climate Dynamics: An Introductory Text. Academic Press, 344 pp.

Mellor, G. L., and T. Ezer, 1995: Sea level variations induced by heating and cooling: An evaluation of the Boussinesq approximation in ocean models. J. Geophys. Res., 100 (C10), $20565-20577$.

Nakada, M., and K. Lambeck, 1987: Glacial rebound and relative sea-level variations: A new appraisal. Geophys. J. Roy. Astron. Soc., 90, 171-224, doi:10.1111/j.1365-246X.1987.tb00680.x.

Oddo, P., M. Adani, N. Pinardi, C. Fratianni, M. Tonani, and D. Pettenuzzo, 2009: A nested Atlantic-Mediterranean Sea general circulation model for operational forecasting. Ocean Sci., 5, 461-473, doi:10.5194/os-5-461-2009.

Pedlosky, J., 1987: Geophysical Fluid Dynamics. 2nd ed. SpringerVerlag, $710 \mathrm{pp}$.

Pinardi, N., and E. Masetti, 2000: Variability of the large scale general circulation of the Mediterranean Sea from observations and modelling: A review. Palaeogeogr. Palaeoclimatol. Palaeoecol., 158, 153-173.

Pujol, M. I., and G. Larnicol, 2005: Mediterranean Sea eddy kinetic energy variability from 11 years of altimetric data. J. Mar. Syst., 58, 121-142, doi:10.1016/j.jmarsys.2005.07.005.

Solomon, S., D. Qin, M. Manning, Z. Chen, M. Marquis, K. Averyt, M. M. B. Tignor, and H. L. Miller Jr., 2007: Climate Change 2007: The Physical Science Basis. Cambridge University Press, 996 pp. 